Article

\title{
Analysis of environmental factors caused by exposure to air pollution in Yeosu industrial complex: Analyzing Google Trend Trends in Cancer Generation through Big Data
}

\author{
Kil Yong Choi ${ }^{1}$, BuSoon Son ${ }^{2}$, WonHo Yang ${ }^{3}$ \\ 1 Department of Environmental Energy Engineering, Anyang University, Anyang-si 14028, Republic of \\ Korea; bestchoi94@anyang.ac.kr (K.Y.C); \\ 2 Department of Environmental Health Science, Soonchunhyang University, Asan-si 31538, Republic of \\ Korea; sonbss@sch.ac.kr (B.S.S.) \\ 3 Department of Occupational Health, Catholic University of Daegu, Gyeongsan-si 38430, Republic of Korea; \\ whyang@cu.ac.kr (W.H.Y.) \\ * Correspondence: whyang@cu.ac.kr; Tel.: +82-53-850-3739, Fax : +82-53-850-3736
}

\begin{abstract}
Depending on the characteristics of the industrial area, toxicity evaluation of human body, risk assessment and health impact assessment may directly cause cancer due to air pollution. Environmental data collection is from August 2018 to January 31, 2019, and the average, minimum, and maximum values of air pollution data respectively. According to the global data on global trends using the Big Data, high blood pressure is confirmed at 33rd place in the world, and myocardial infarction among the environmental diseases is confirmed to be lower than Korea. Disease that occurred in Jeolla province industrial complex considering the characteristics of our country was identified as representative. Air pollutants are considered to be the causes of allergic diseases in Korea. PM10 was found to be higher than the control area $\left(28.8804348\left(\mu \mathrm{g} / \mathrm{m}^{3}\right)\right.$, $31.7065217\left(\mu \mathrm{g} / \mathrm{m}^{3}\right)$ and $32.8532609\left(\mu \mathrm{g} / \mathrm{m}^{3}\right)$. The mean concentrations of PM2.5 in the middle and high exposure areas were lower than those of the control areas, but the highest in the intermediate exposure areas was $16.5978261\left(\mu \mathrm{g} / \mathrm{m}^{3}\right), 16.1086957\left(\mu \mathrm{g} / \mathrm{m}^{3}\right)$ and $17.1847826(\mu \mathrm{g} /$ $\mathrm{m}^{3}$ ) respectively. The relationship between the major variables of environmental exposure in Yeosu was confirmed to be correlated with high blood pressure, chronic obstructive pulmonary disease (COPD), bronchitis, cerebrovascular, diabetes, thyroid disease, sinus infection, anemia and pneumonia.
\end{abstract}

Keywords: Google Trend; Particulate Matter; National Ambient Air Quality Monitoring Information System; Chronic obstructive pulmonary disease; Big Data

\section{Introduction}

In Korea, diseases caused by the environment exposed to the occupants in the industrial complex area can occur. This has been found to affect exposure of secondary pollutants through diffusion of the atmosphere $[1,2]$. Depending on the characteristics of the industrial area, toxicity evaluation of human body, risk assessment and health impact assessment may directly cause cancer due to air pollution $[3,4]$. Previous studies of the past have shown that air pollution directly affects lung disease and respiratory diseases in the United States in 1948, a case of air pollution in Pennsylvania, USA $[5,6,7]$. In Korea, it has been confirmed that the application of industrial deterrence (Yeosu, Gwangyang, Ulsan, Pohang, Ansan) has increased as a factor of cancer occurrence and disease in recent years through the process of establishing industrial complexes in major economic 
development since $1970[8,9]$ Among them, heavy metals and chemicals in Korea are identified as air pollutants, and fine dust due to fuel consumption is also confirmed [10,11]. As a result, the emission factors of odor, air pollution, soil pollution, and heavy metals directly affect the residents of the area due to the composition of the industrial complex. Through these results, it becomes a socially very serious environmental impact. Based on this, epidemiological studies have been actively conducted in Korea, and the characteristics of this study are to provide a management method to reduce the possibility of exposure to environmental pollutants considering regional characteristics $[12,13]$. It is necessary to investigate the health damage caused by exposure to environmental pollutants in the industrial complex area using BIG DATA, and to identify the cause of cancer in residents of exposed areas [14, 15]. Therefore, it is necessary to confirm whether the exposure from environmental pollution directly affects the health effect in the industrial complex area $[16,17,18]$, and it is necessary to use the BIG DATA as the basis of various studies for the purpose of environmental health for evaluation. This study has been actively carried out in Korea recently to develop the major factors by grasping the magnitude and severity of health impacts and grasping pathways for exposure to environmental harmful factors [19]. I would like to identify the cause of long-term exposure by using direct treatment of cancer occurrence and hospital treatment by checking with Google trend through exposure data in the area.

\section{Materials and methods}

\subsection{Search term (GT, Google Trend)}

As an important indicator of this study, environmental data were collected by focusing on "Percentage Per Day in the Year of 2018". Based on previous research data, we searched based on multi-disciplinary research methodology with expert (e.g., Clinical specialists, epidemiologists, research methodologists and statisticians) knowledge. The search was done in a quantitative way to minimize reliability and risk. According to the global data on global trends using the Big Data, high blood pressure is confirmed at 33rd place in the world, and myocardial infarction among the environmental diseases is confirmed to be lower than Korea. Based on this search, we will examine the tendency of cancer diagnoses in KOREA (Figure 1).

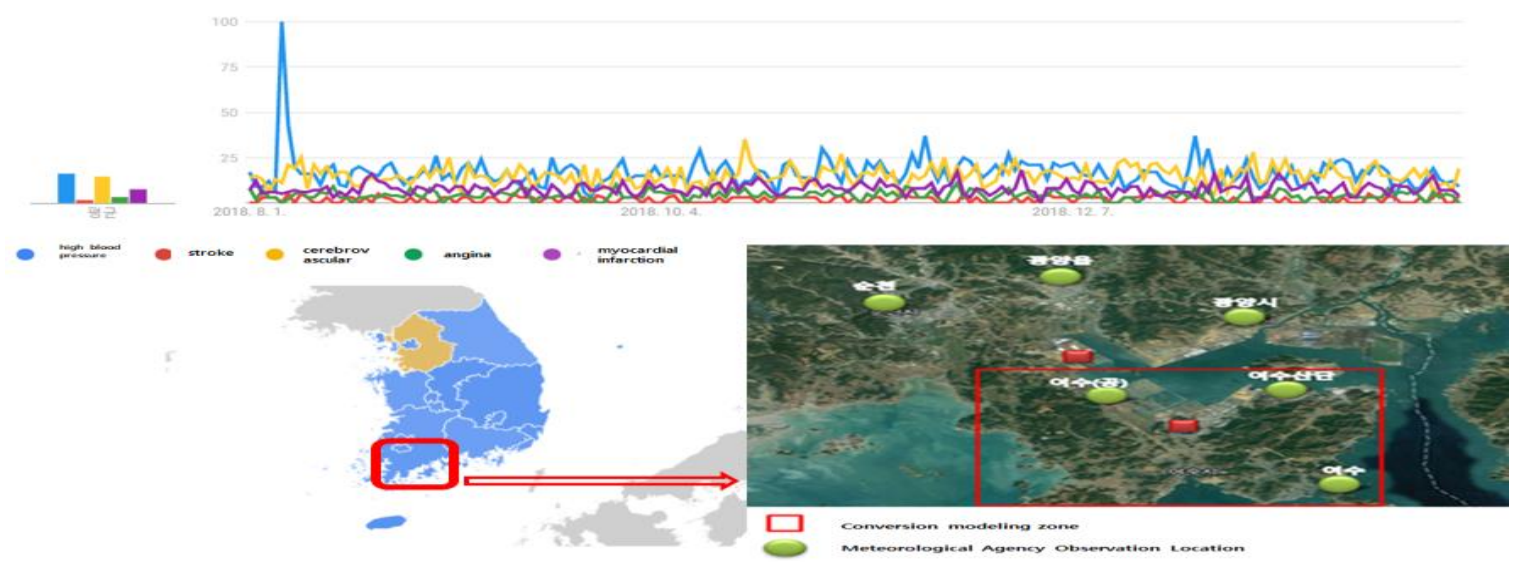

Fig. 1 Comparisons of global Google Trends (GT) and environmental concerns with countries

\subsection{Air pollution measurement data}

The air pollution concentration data of the measuring station were measured as the air pollution monitoring network data operated by the national and local governments. In this study, the measured values of fine dust (PM10, PM2.5) annual mean value (less than $50 \mu \mathrm{g} / \mathrm{m}^{3}$, less than $25 \mu \mathrm{g} / \mathrm{m}^{3}$ ) and 24h average (less than $100 \mu \mathrm{g} / \mathrm{m}^{3}$, less than $50 \mu \mathrm{g} / \beta$-Ray Absorption Method). This method captures suspended particulate matter suspended in the air on a filter paper for a 
certain period of time, permeates the betaine, and continuously measures the weight concentration of the particulate matter.

The sampling flow rate should be constantly inhaled at a set flow rate of 16.7 liters per minute for the correct separation of particulate matter and the flow rate (15.0 liters per minute or less or 18.4 liters per minute or more) The sampling should be discontinued and the sampler checked. The concentration data measured at the measuring station were analyzed using the information gathered by the National Ambient Air Quality Monitoring Information System (NAMIS) based on the Internet.

\subsection{Statistical analysis}

Statistical analysis was done using the statistical program SAS 9.4 and the log value was taken for the normality test of the concentration distribution of air pollution in the search of Google Trends. After confirming the normal value the geometric mean (GM) and geometric standard deviation (GSD). We confirmed the correlation of environmental concentration values by checking the average search data of Google Trends for environmental diseases (e.g., high blood pressure, COPD: chronic obstructive pulmonary disease, asthma) on a daily basis. The t-test was conducted by applying environmental variables and continuous variables (disease of Google Trends).

\section{Results}

\subsection{PM10 concentration in air pollution in Korea}

The disease (e.g., Major diseases in the region: high blood pressure, stroke, cerebrovascular, angina, myocardial infarction, diabetes, anemia, COPD: chronic obstructive pulmonary disease, bronchitis) that occurred in Jeolla province industrial complex considering the characteristics of our country was identified as representative. Air pollutants are considered to be the causes of allergic diseases in Korea. It was found that the concentration of airborne pollutants was slightly higher in the high and medium exposure areas than in the control areas of the substance. Figure 2 shows that the environmental data collection is from August 2018 to January 31, 2019, and the average, minimum, and maximum values of air pollution data respectively.

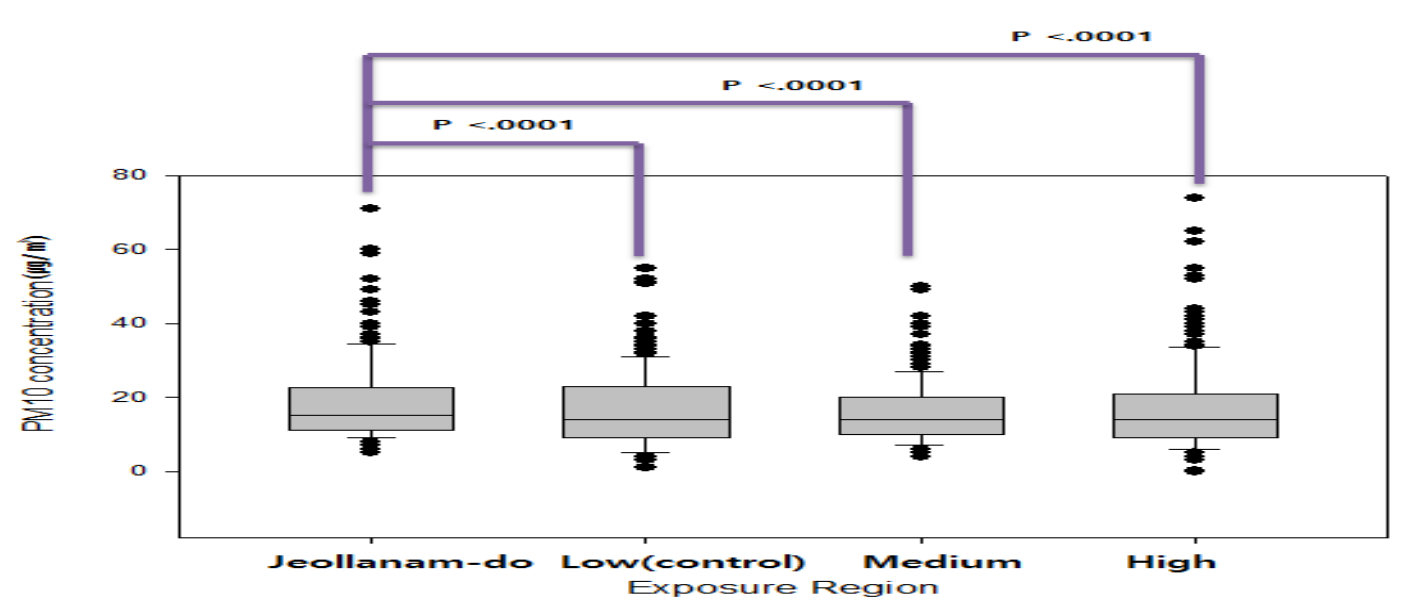

Fig. 2 Analysis of Correlation between Low, Medium and High Exposure (PM10) Areas in Jeollanam - d

o using Air Korea Data

Table 1 shows the results of checking the exposure area of the environment through the indicators of environmental data. Compared with the control area (Yulchon-myeon), the mean values of the medium (e.g., Myongdong-dong, Dundeok-dong, Huyang-myeon and Yeochun-dong) and high exposure areas (e.g., Samil-dong and Haesan-dong) were statistically significant. Compared to the control area, the PM10 (Particulate Matter) was found to be higher than the 
control area $\left(28.8804348\left(\mu \mathrm{g} / \mathrm{m}^{3}\right), 31.7065217\left(\mu \mathrm{g} / \mathrm{m}^{3}\right)\right.$ and $32.8532609\left(\mu \mathrm{g} / \mathrm{m}^{3}\right)$. The mean concentrations of PM2.5 in the middle and high exposure areas were lower than those of the control areas, but the highest in the intermediate exposure areas was $16.5978261\left(\mu \mathrm{g} / \mathrm{m}^{3}\right), 16.1086957(\mu \mathrm{g} /$ $\left.\mathrm{m}^{3}\right)$ and $17.1847826\left(\mu \mathrm{g} / \mathrm{m}^{3}\right)$ respectively.

Table 1 Measurement of outdoor air pollution concentration using Air Korea data

\begin{tabular}{lcccc}
\hline & N & Minimum & Maximum & Average \\
\hline $\mathbf{P M}_{10}\left(\mu \mathrm{g} / \mathrm{m}^{3}\right)^{*}$ & 184 & 28.8804348 & 6 & 117 \\
$\mathbf{P M}_{2.5}\left(\mu \mathrm{g} / \mathrm{m}^{3}\right)^{*}$ & 184 & 16.5978261 & 1 & 55 \\
$\mathbf{P M}_{10}\left(\mu \mathrm{g} / \mathrm{m}^{3}\right)^{* *}$ & 184 & 31.7065217 & 8 & 114 \\
$\mathbf{P M}_{2.5}\left(\mu \mathrm{g} / \mathrm{m}^{3}\right)^{* *}$ & 184 & 16.1086957 & 4 & 50 \\
$\mathbf{P M}_{10}\left(\mu \mathrm{g} / \mathrm{m}^{3}\right)^{* * *}$ & 184 & 32.8532609 & 8 & 114 \\
$\mathbf{P M}_{2.5}\left(\mu \mathrm{g} / \mathrm{m}^{3}\right)^{* * *}$ & 184 & 17.1847826 & 0 & 74 \\
\hline
\end{tabular}

${ }^{*}$ Low (control) exposure region, ${ }^{* *}$ Medium exposure region, ${ }^{* * *}$ High exposure region

\section{2. $\mathrm{PM}_{2.5}$ concentration in air pollution in Korea}

The results of analyzing air pollution according to the characteristics of the industrial complex in Korea were confirmed. This confirmed the risk of air pollution in Jeollanam-do due to the high exposure area after the fine dust was designated as a special law by the National Institute of Environmental Science. In addition, it is necessary to confirm the cancer diagnosis data of the disease and to discuss ways to manage it postoperatively. This will require management according to the characteristics of Korea's industrial complex, and the overall concentration of Jeolla-do was statistically significant in low, medium, and high concentrations. Considering these points in the future, the high concentration of Jeolla Province should be managed. This is thought to be the cause of cancer in the long term, and may affect even chronic diseases through management.

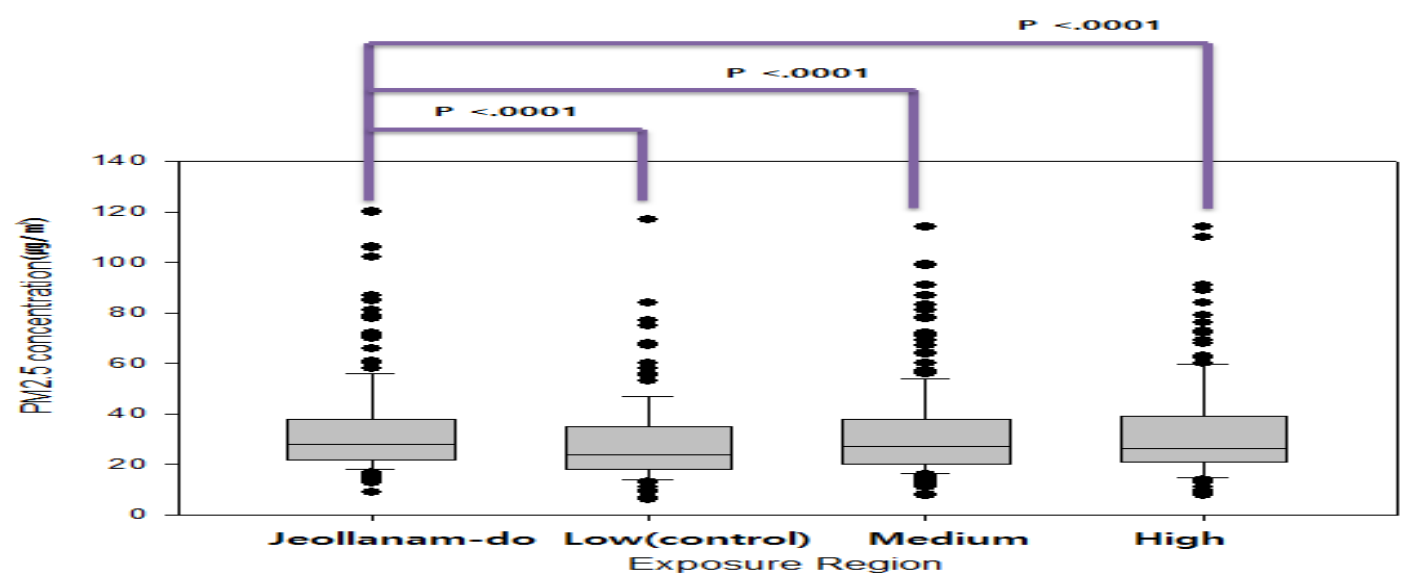

Fig. 3 Analysis of Correlation between Low, Medium and High Exposure (PM2.5) Areas in Jeollanam do using Air Korea Data.with countries

Table 2 shows the results of correlation analysis between air pollution data (atmospheric environment) and diseases are as follows. In PM10, medium (1.15(1.07-1.23)) and high (1.99(1.97-2.01)) exposure were statistically significant, respectively, compared to low exposure. Even in PM2.5, medium (1.20(1.11-1.30)) and high (1.93(1.88-1.98)) exposure were statistically significant, respectively, compared to low exposure. High exposure areas were higher than low exposure and medium exposure.

Table 2 Analysis of Correlation between Air Pollution Data (atmospheric environment) and Disease 


\begin{tabular}{|c|c|c|c|c|}
\hline control region & Mean & \multicolumn{2}{|c|}{ 95\% CL Mean } & $\operatorname{Pr}>|t|$ \\
\hline Medium exposure area ${ }^{* * * *}$ & 1.1486 & 1.0657 & 1.2316 & \multirow{2}{*}{$<.0001^{* * *}$} \\
\hline High exposure area ${ }^{* * *}$ & 1.9911 & 1.9734 & 2.0088 & \\
\hline Medium exposure area $a^{* * * * *}$ & 1.2027 & 1.1089 & 1.2965 & \multirow{2}{*}{$<.0001^{* * *}$} \\
\hline High exposure area ${ }^{* * * *}$ & 1.9286 & 1.8801 & 1.977 & \\
\hline
\end{tabular}

${ }^{*} P<0.05 ;{ }^{* *} P<0.01 ;{ }^{* * *} P<0.001$

**** control region, Medium exposure area, High exposure area

***** control region, Medium exposure area, High exposure area

\subsection{Correlation between Air Pollution and Disease}

As shown in Table 3, the relationship between PM10, which is fine dust in environmental data, and disease was confirmed as follows. As a result, high blood pressure (Higher Region Mean =20.3, 95\% CL Mean = 18.6-22.1), COPD (Higher Region Mean $=24.4,95 \%$ CL Mean = 20.5-28.3) and pneumonia (Higher Region Mean $=40.4,95 \%$ CL Mean $=37.2-43.6)$ were found to be statistically correlated in the control area $(P=0.0286,0.0437,0.044)$. And high blood pressure (Higher Region Mean $=20.4,95 \% C L$ Mean $=$ 18.7-22.1), bronchitis (Higher Region Mean =19.6, 95\% CL Mean =15.6-23.6), and pneumonia (Higher Region Mean $=40.3,95 \%$ CL Mean $=37.3-43.3)$ were found to be statistically correlated in the intermediate exposure area $(P=0.0227,0.0487,0.0176)$. Finally, in high exposure areas, cerebrovascular (Higher Region Mean $=21.7,95 \%$ CL Mean $=18.5-25.0$ ), diabetes (Higher Region Mean $=48.9,95 \%$ CL Mean $=46.7-51.0)$, thyroid disease (Higher Region Mean =33.6, 95\% CL Mean =30.7-36.5), bronchitis (Higher Region Mean $=19.2,95 \%$ CL Mean $=15.2-23.1$ ), and pneumonia (Higher Region Mean $=40.1,95 \%$ CL Mean $=37.0-43.2)$ were found to be statistically correlated with each other $(P=0.0531,0.001,0.0121,0.033,0.0109)$.

Table 3 Analysis of Correlation between Air Pollution Data $\left(\mathrm{Pm}_{10}, \mu \mathrm{g} / \mathrm{m}^{3}\right)$ and Disease

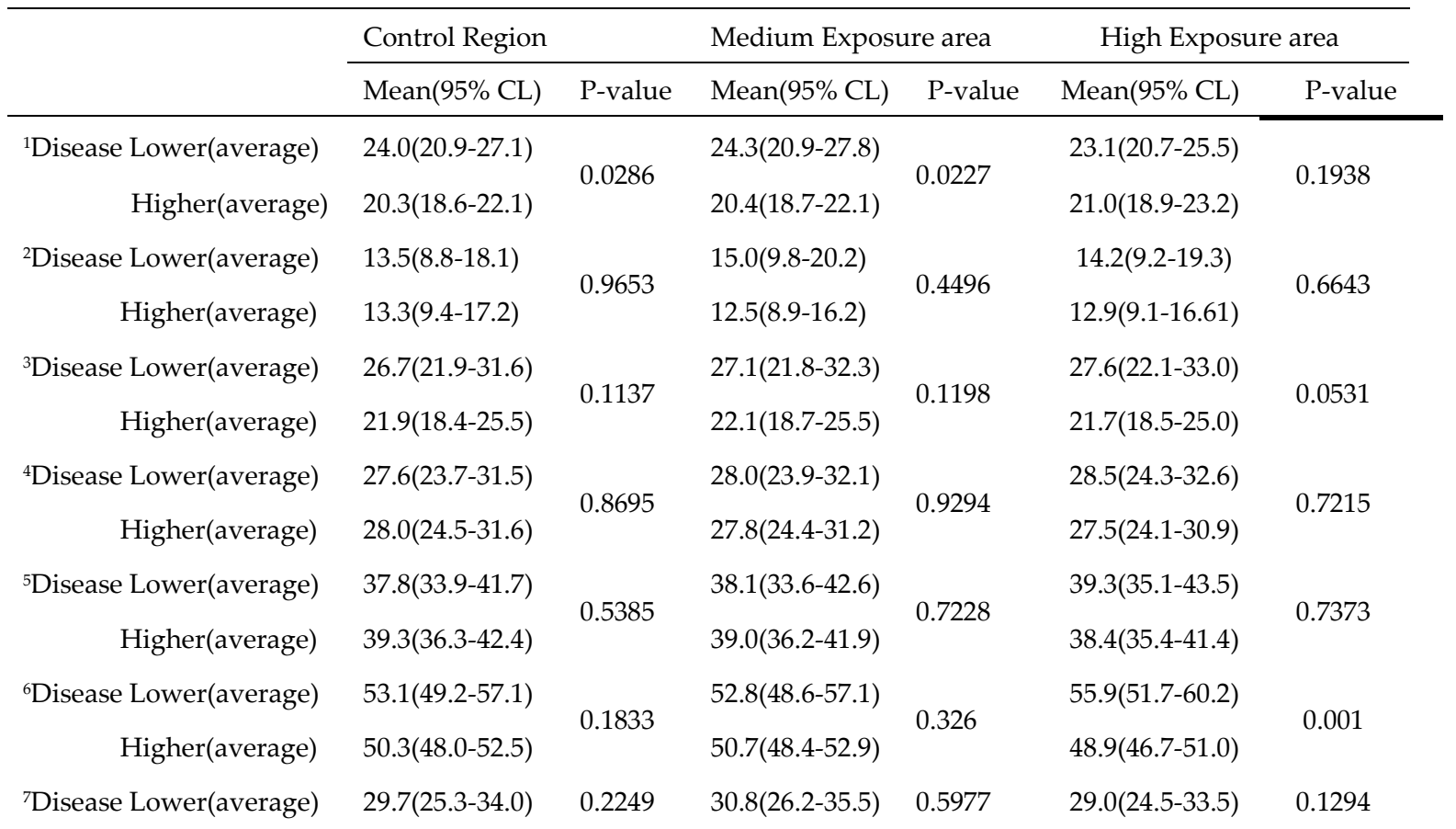




\begin{tabular}{|c|c|c|c|c|c|c|}
\hline Higher(average) & $33.3(29.3-37.4)$ & & $32.4(28.6-36.3)$ & & $33.5(29.6-37.4)$ & \\
\hline${ }^{8}$ Disease Lower(average) & $38.4(33.8-43.1)$ & \multirow{2}{*}{0.1291} & $38.9(33.7-44.1)$ & \multirow{2}{*}{0.1097} & $40.3(35.5-45.2)$ & \multirow{2}{*}{0.0121} \\
\hline Higher(average) & $34.4(31.5-37.4)$ & & $34.5(31.7-37.3)$ & & $33.6(30.7-36.5)$ & \\
\hline Disease Lower(average) & $31.0(25.8-36.2)$ & \multirow{2}{*}{0.0437} & $27.9(22.7-33.2)$ & \multirow{2}{*}{0.6656} & $29.3(23.7-34.8)$ & \multirow{2}{*}{0.2993} \\
\hline Higher(average) & $24.4(20.5-28.3)$ & & $26.5(22.6-30.5)$ & & 25.7(21.9-29.6) & \\
\hline${ }^{10}$ Disease Lower(average) & 25.7(20.1-31.3) & \multirow{2}{*}{0.0874} & $26.7(20.5-33.0)$ & \multirow{2}{*}{0.0487} & $27.2(20.9-33.4)$ & \multirow{2}{*}{0.033} \\
\hline Higher(average) & 19.6(15.4-23.9) & & $19.6(15.6-23.6)$ & & $19.2(15.2-23.1)$ & \\
\hline${ }^{11}$ Disease Lower(average) & $17.4(11.6-23.3)$ & \multirow{2}{*}{0.954} & $16.5(10.2-22.8)$ & \multirow{2}{*}{0.7436} & $17.1(10.9-23.2)$ & \multirow{2}{*}{0.9215} \\
\hline Higher(average) & $17.2(13.0-21.4)$ & & $17.7(13.6-21.8)$ & & $17.4(13.3-21.5)$ & \\
\hline 12Disease Lower(average) & $14.4(9.8-19.0)$ & \multirow{2}{*}{0.8344} & $12.4(7.8-16.9)$ & \multirow{2}{*}{0.4652} & $15.3(10.5-20.1)$ & \multirow{2}{*}{0.507} \\
\hline Higher(average) & 13.7(9.5-17.9) & & 14.8(10.7-18.9) & & 13.2(9.2-17.2) & \\
\hline${ }^{13}$ Disease Lower(average) & $28.5(23.0-33.9)$ & \multirow{2}{*}{0.5047} & $30.4(24.2-36.6)$ & \multirow{2}{*}{0.1364} & $28.7(23.2-34.2)$ & \multirow{2}{*}{0.4629} \\
\hline Higher(average) & $26.2(22.2-30.1)$ & & $25.3(21.7-29.0)$ & & $26.2(22.2-30.1)$ & \\
\hline${ }^{14}$ Disease Lower(average) & $4.4(1.2-7.6)$ & \multirow{2}{*}{0.3043} & $4.3(0.9-7.8)$ & \multirow{2}{*}{0.3731} & $4.8(0.7-8.9)$ & \multirow{2}{*}{0.5202} \\
\hline Higher(average) & $6.8(3.4-10.3)$ & & 6.7(3.4-9.9) & & $6.5(3.4-9.5)$ & \\
\hline${ }^{15}$ Disease Lower(average) & 16.8(11.9-21.8) & \multirow{2}{*}{0.2543} & 16.7(11.3-22.2) & \multirow{2}{*}{0.3354} & $16.9(11.3-22.5)$ & \multirow{2}{*}{0.2998} \\
\hline Higher(average) & 13.3(9.6-17.0) & & 13.6(10.1-17.1) & & 13.5(10.1-16.9) & \\
\hline${ }^{16}$ Disease Lower(average) & $20.5(15.6-25.4)$ & \multirow{2}{*}{0.9112} & 22.7(17.4-28.1) & \multirow{2}{*}{0.2639} & 21.5(16.4-26.5) & \multirow{2}{*}{0.5726} \\
\hline Higher(average) & 20.1(16.0-24.3) & & 19.0(15.1-22.9) & & 19.6(15.6-23.6) & \\
\hline${ }^{17}$ Disease Lower(average) & $8.8(4.3-13.3)$ & \multirow{2}{*}{0.697} & $6.1(2.0-10.2)$ & \multirow{2}{*}{0.3004} & $8.0(3.6-12.5)$ & \multirow{2}{*}{0.9444} \\
\hline Higher(average) & 7.7(4.0-11.3) & & $9.2(5.5-12.9)$ & & $8.2(4.6-11.8)$ & \\
\hline${ }^{18}$ Disease Lower(average) & $8.8(4.3-13.3)$ & \multirow{2}{*}{0.697} & $6.1(2.0-10.2)$ & م 200 & $8.0(3.6-12.5)$ & \\
\hline Higher(average) & 7.7(4.0-11.3) & & $9.2(5.5-12.9)$ & & $8.2(4.6-11.8)$ & \\
\hline${ }^{19}$ Disease Lower(average) & $45.8(41.6-50.1)$ & & $46.9(42.3-51.5)$ & & $47.0(42.6-51.4)$ & \\
\hline Higher(average) & $40.4(37.2-43.6)$ & & 40.3(37.3-43.3) & & $40.1(37.0-43.2)$ & \\
\hline${ }^{20}$ Disease Lower(average) & 2.7(-1.1-6.5) & & 3.1(-1.3-7.5) & & $3.0(-1.2-7.2)$ & \\
\hline Higher(average) & $1.6(-0.6-3.9)$ & & $1.5(-0.6-3.6)$ & & $1.5(-0.6-3.7)$ & \\
\hline${ }^{21}$ Disease Lower(average) & $14.6(8.6-20.7)$ & & $12.5(6.4-18.5)$ & & $14.0(8.0-20.0)$ & \\
\hline Higher(average) & 12.5(8.5-16.6) & & 13.8(9.7-18.0) & & $13.0(8.9-17.2)$ & \\
\hline
\end{tabular}

${ }^{1}$ High blood pressure, ${ }^{2}$ Stroke, ${ }^{3}$ Cerebrovascular, ${ }^{4}$ Angina, ${ }^{5}$ Myocardial infarction, ${ }^{6}$ Diabetes, ${ }^{7}$ Anemia, ${ }^{8}$ Thyroid disease, ${ }^{9} \mathrm{COPD}$, chronic obstructive pulmonary disease, ${ }^{10}$ Bronchitis, ${ }^{11}$ Pulmonary emphysema, ${ }^{12}$ Bronchiectasis, ${ }^{13}$ Asthma, ${ }^{14}$ Sinus infection, ${ }^{15}$ Allergic coryza, ${ }^{16}$ Allergy desmatitis, ${ }^{17}$ Bronchial asthma, ${ }^{18}$ Acute bronchitis, ${ }^{19}$ Pneumonia, ${ }^{20}$ Acute pneumonia, ${ }^{21}$ Skin Disease

Table 4 shows the relationship between PM2.5, which is fine dust in environmental data, and disease. In contrast, high blood pressure (Higher Region Mean =20.1, 95\% CL Mean = 18.5-21.8), COPD (Higher Region Mean =23.8, 95\% CL Mean =20.1-27.5), sinus infection (Higher Region Mean =7.9, 95\% CL Mean $=4.2-11.5$ ), and pneumonia (Higher Region Mean $=40.3,95 \%$ CL Mean $=37.1-43.4$ ) were found to be statistically correlated with each other $(P=0.0157,0.019,0.0518,0.035)$. High-blood pressure, cerebrovascular, anemia, and pneumonia were found to be statistically correlated in the middle-exposed areas. High blood pressure, cerebrovascular, thyroid disease, COPD and pneumonia were found to be statistically correlated in the high exposure area.

Table 4 Analysis of Correlation between Air Pollution Data $\left(\operatorname{Pm} 2.5, \mu \mathrm{g} / \mathrm{m}^{3}\right)$ and Disease 


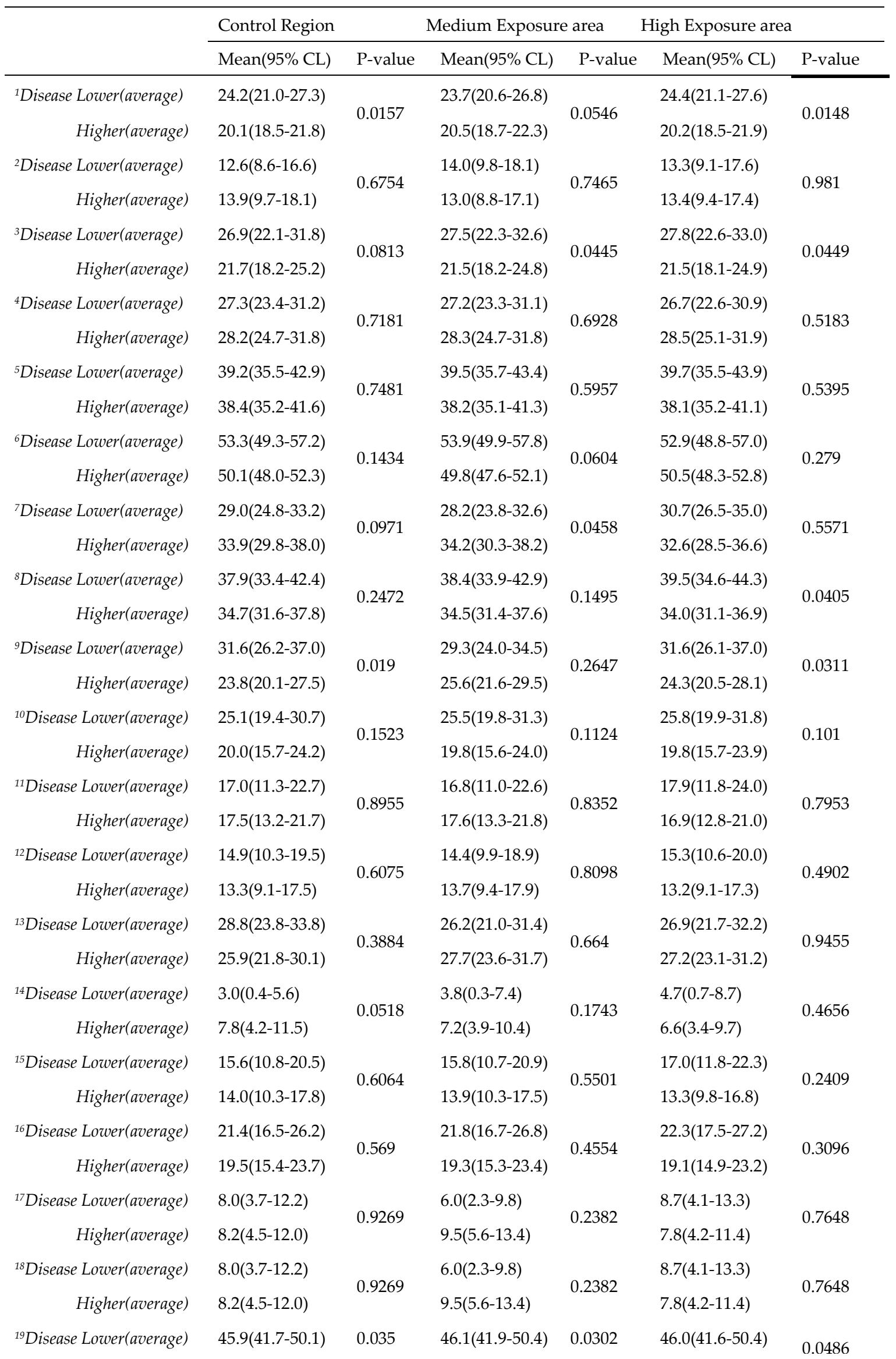




\begin{tabular}{rllllll} 
Higher(average) & $40.3(37.1-43.4)$ & & $40.3(37.1-43.4)$ & & $40.6(37.5-43.7)$ & \\
${ }^{20}$ Disease Lower(average) & $2.6(-1.1-6.3)$ & & $2.7(-1.1-6.6)$ & & $2.9(-1.2-7.0)$ & 0.5261 \\
Higher(average) & $1.6(-0.7-4.0)$ & 0.6389 & $1.6(-0.6-3.9)$ & & $1.6(-0.6-3.7)$ & \\
${ }^{21}$ Disease Lower(average) & $16.0(9.8-22.3)$ & & $13.5(7.6-19.4)$ & & $15.1(8.4-21.7)$ & 0.4458 \\
Higher(average) & $11.5(7.7-15.4)$ & 0.2003 & $13.3(9.1-17.4)$ & & $12.4(8.6-16.1)$ & \\
\hline
\end{tabular}

${ }^{1}$ High blood pressure, ${ }^{2}$ Stroke, ${ }^{3}$ Cerebrovascular, ${ }^{4}$ Angina, ${ }^{5}$ Myocardial infarction, ${ }^{6}$ Diabetes, ${ }^{7}$ Anemia, ${ }^{8}$ Thyroid disease, ${ }^{9} \mathrm{COPD}$, chronic obstructive pulmonary disease, ${ }^{10}$ Bronchitis, ${ }^{11}$ Pulmonary emphysema, ${ }^{12}$ Bronchiectasis, ${ }^{13}$ Asthma, ${ }^{14}$ Sinus infection, ${ }^{15}$ Allergic coryza, ${ }^{16}$ Allergy desmatitis, ${ }^{17}$ Bronchial asthma, ${ }^{18}$ Acute bronchitis, ${ }^{19}$ Pneumonia, ${ }^{20}$ Acute pneumonia, ${ }^{21}$ Skin Disease

\section{Discussion}

This study was carried out based on BIG DATA for the evaluation of exposure due to environmental pollution in industrial area in recent 1 years. In the case of residential areas for high-exposure areas, middle-exposure areas and low-exposure areas (comparison) of Yeosu in the background of industrial complexes under study, the risk of cancer development including death in medium and high Was confirmed to be high. As a result, consistent disease according to the characteristics of Yeosu area according to all types of cancer will occur and follow up of the disease will be necessary. According to the recent data of Yeosu, the rate of housing distribution has been confirmed as $99.3 \%$. As a result, considering the special features of oil refinery and petrochemical formation in Yeosu National Industrial Complex, It can be confirmed that the diffusion due to pollution is damaged in some areas in the natural environment [20,21]. In this study, it was also confirmed that the external factors related to the disease are affecting climate change and global warming. The aim of this study was to evaluate the effects of exposure to air pollution on 21 outbreaks of environmental diseases including "High blood pressure, Stroke, Cerebrovascular, Angina, Myocardial infarction, Diabetes, Anemia, Thyroid disease, Chronic obstructive pulmonary disease: COPD, Bronchitis, Pulmonary emphysema, Acute bronchitis, Acute pneumonia, and Skin Disease "were identified in patients with Alzheimer 's disease, Asthma, Sinus infection, Allergic coryza, Allergy desmatitis, Bronchial asthma, Acute bronchitis. This disease was mainly searched and the GT (Google Trends) was used in Korea and international case study, and the systematic review of the search was conducted without any problems $[22,23]$. The second was that the disease was exposed to the Korean environment and it was thought that the value as BIG DATA would be increased. Exposure to environmentally hazardous substances is a major variable and should be identified as a major factor in future disease management. As a result of confirming the air quality of the industrial complex in Cholla province where the representative pollutants of Korea are generated, the results of the preliminary analysis of the health index of the target area and the affinity of the health of the urban environment suggests a way to construct the health city of Yeosu , But the analysis of the indicators using BIG data seems to be very poor. In this study, daily mean values were calculated using trend data of PM10 and PM2.5 measured in the air pollution monitoring network in Korea. The data were applied to the requirements of the analysis according to the weather conditions using the generalized linear model, And concentration characteristics of industrial complex in Jeolla province were analyzed. Although there is no limit to the limit of Google Trends, the quality of air collected by the National Institute of Environmental Research (AIR KOREA) has been analyzed since August, 2018, so it is possible to use data for about one year recently. And the relationship between exposure to air and chronic diseases and some diseases. Compared with the control area, the high tendency of air pollution was found to be correlated with some diseases in the medium exposure area and the high exposure area. According to the results of previous studies, the correlation between air pollutants, environmental diseases such as atopic dermatitis and asthma disease has been reported. Based on this, it will be necessary to use BIG DATA for further verification. As a result of completing the primary screening check, it is necessary 
to confirm the relationship between exposure to environmental pollution and environmental pollution. The relationship between PM10, which is a fine dust in the environmental data, and disease was confirmed to be correlated with high blood pressure and COPD and Pneumonia in the control area. High blood pressure, bronchitis, and pneumonia were found to be correlated in the intermediate exposure area. Cerebrovascular, Diabetes, Thyroid disease, Bronchitis and Pneumonia were found to be correlated in high exposed areas. The relationship between PM2.5, which is the fine dust in the environmental data, and the disease, was confirmed to be correlated with high blood pressure, COPD, sinus infection, and pneumonia in the control area. High blood pressure, Cerebrovascular, Anemia, and Pneumonia were found to be correlated in the intermediate exposure area. In high exposed areas, high blood pressure, cerebrovascular, thyroid disease, and chronic obstructive pulmonary disease (COPD) and pneumonia were found to be correlated. This study was conducted to confirm the correlation between air pollutants and exposure of the disease and to reduce the time by controlling, matching, and correcting disturbance variables through previous studies. Because of the selective bias, we were able to confirm the importance of this study.

Conclusion, the results show that there is a limit to the information based on some data that affect life style or health that can be obtained based on 21 major variables of exposure of environment designated by NESI (National Environmental Science Institute), Analyzing the regional impacts and personal factors of women has been limited. There are very few researches to produce these research results in Korea, and it is necessary to use them as appropriate indicators in consideration of the regional characteristics of the infrastructure. The relationship between the major variables of environmental exposure in Yeosu was confirmed to be correlated with high blood pressure, chronic obstructive pulmonary disease (COPD), bronchitis, cerebrovascular, diabetes, thyroid disease, sinus infection, anemia and pneumonia. Therefore, the results obtained through the analysis of this study are meaningful in that they can present directions for future research rather than their own interpretation. It is expected that it will be used as a basic data when conducting studies to evaluate the health effects of exposure to industrial pollution using secondary data. Therefore, in order to investigate further precise epidemiological studies, we conducted a linkage study on environmental pollution data and health insurance data for major diseases that have been found to be significantly related to environmental exposures in industrial complexes or industrial complexes that are related to industrial pollution. You need to do it.

Acknowledgments: The study was approved by Soonchunhyang University institutional review board "Title : Monitoring of Environmental Pollutants Exposure and Health Effects in Industrial Residents(Gwangyang $Y$ eosu, 3nd Phase, 1st Year)" (IRB no. 1040875-201807-BR-028).

List of abbreviations: COPD: chronic obstructive pulmonary disease; GT: Google Trend; PM: Particulate Matter; GM: geometric mean; GSD: geometric standard deviation; NAMIS: National Ambient Air Quality Monitoring Information System; NESI: National Environmental Science Institute; CI confidence interval.

Availability of data and materials: The datasets analyzed in the current study are available from the corresponding author upon reasonable request.

\section{References}

1. Nam, D. H., LEE, D. P., 2006. Monitoring for $\mathrm{Pb}$ and $\mathrm{Cd}$ pollution using feral pigeons in rural, urban, and industrial environments of Korea. Science of the Total Environment 357(1-3), 288-295.

2. Park, H. j., Lee, K. H., Moon, C. S., Woo, K. S., Kang, T. S., Chung, E. K., Son, B. S., 2015. Simultaneous exposure to heavy metals among residents in the industrial complex: Korean national cohort study. International journal of environmental research and public health 12(6), 5905-5917.

3. HERTWICH, E. G., PEASE, W. S., MCKONE, T. E., 1998. Environmental Policy Analysis: Evaluating Toxic 
Impact Assessment Methods: What Works Best. Environmental science \& technology 32(5), 138A-144A.

4. LANDRIGAN, P. J., Kimmel, C. A., Correa, A., Eskenazi, B., 2004. Children's health and the environment: public health issues and challenges for risk assessment. Environmental health perspectives 112(2), 257-265.

5. Gilliland, F. D., McConnell, R., Peters, J., Jr, H. G., 1999. A theoretical basis for investigating ambient air pollution and children's respiratory health. Environmental health perspectives 107(3), 403-407.

6. Joel, S., 1994. What are people dying of on high air pollution days. Environmental research 64(1), 26-35.

7. DOCKERY, D. W., SCHWARTZ, J., SPENGLER, J. D., 1992. Air pollution and daily mortality: associations with particulates and acid aerosols. Environmental research 59(2), 362-373.

8. CHO, S. H., KANG, D. H., KO, K. S., KWON, H. J., KIM, D. H., HA, M., HAN, S. H., JU, Y. S., 1997. Estimates of occupational cancer in Korea. Journal of occupational health 39(3), 192-196.

9. Cho, J. Y., Son, J. G., Park, B. J., Chung, B. Y., 2009. Distribution and pollution sources of polycyclic aromatic hydrocarbons (PAHs) in reclaimed tidelands and tidelands of the western sea coast of South Korea. Environmental monitoring and assessment 149(1-4), 385-393.

10. Kim, K. H., Choi, G. H., Kang, C. H., Lee, J. H., Kim, J. Y., Youn, Y. H., Lee, S. R., 2003. The chemical composition of fine and coarse particles in relation with the Asian Dust events. Atmospheric Environment 37(6), 753-765.

11. Choung, S. W., Oh, J. S., Han, W. S., Chon, C. M., Kwon, Y. S., Kim, D. Y., Shin, W. S., 2016. Comparison of physicochemical properties between fine (PM2. 5) and coarse airborne particles at cold season in Korea. Science of the Total Environment 541, 1132-1138.

12. Cole, M. A., Elliott, R. J. R., Shimamoto, K., 2005. Industrial characteristics, environmental regulations and air pollution: an analysis of the UK manufacturing sector. Journal of environmental economics and management 50(1), 121-143.

13. Bell, M. L., Cifuentes, L. A., Davis, D. L., Cushing, E., Telle, A. G., Gouvei, N., 2011. Environmental health indicators and a case study of air pollution in Latin American cities. Environmental research 111(1), 57-66.

14. Chen, X., Shao, S., Tian, Z., Xie, Z., Yin, P., 2017. Impacts of air pollution and its spatial spillover effect on public health based on China's big data sample. Journal of cleaner production 142, 915-925.

15. Chen, M., Yang, J., Hu, L., Hossain, M. S., Muhammad, G., 2018. Urban healthcare big data system based on crowdsourced and cloud-based air quality indicators. IEEE Communications Magazine 56(11), 14-20.

16. Bergstr, A. D., Brunekreef, B., Burdorf, A., 2018. The effect of industry-related air pollution on lung function and respiratory symptoms in school children. Environmental Health 17(1), 30.

17. Torres, P., Ferreira, J., Monteiro, A., Costa, S., Pereira, M. C., Madureira, J., Mendes, A., Teixeira, J. P., 2018. Air pollution: A public health approach for Portugal. Science of the total environment 643, 1041-1053.

18. Chang, T. Y., Zivin, J. G., Gross, T., Neidell, M., 2019. The Effect of Pollution on Worker Productivity: Evidence from Call Center Workers in China. American Economic Journal: Applied Economics 11(1), 151-72.

19. Wang, Y., Kung, L. A., Wang, W. Y. C., Cegielski, C. G.. 2018. An integrated big data analytics-enabled transformation model: Application to health care. Information \& Management 55(1), 64-79.

20. Won, Y. J., Jung, K. W., Oh, C. M., Park, E. H., Kong, H. J., Lee, D. H., Lee, K. H., 2018. Geographical Variations and Trends in Major Cancer Incidences throughout Korea during 1999-2013. Cancer research and treatment: official journal of Korean Cancer Association 50(4), 1281.

21. Hong, E. J., Lee, S. W., Kim, G. B., Kim, T. J., Kim, H. W., Lee, K. H., Son, B. S., 2018. Effects of Environmental Air Pollution on Pulmonary Function Level of Residents in Korean Industrial Complexes. International journal of environmental research and public health 15(5), 834.

22. Faridi, S., hamsipour, M., Krzyzanowski, M., Künzli, N., Amini, H., Azimi, F., Malkawi, M., Momeniha, F., Gholampour, A., Hassanvand, M. S., Naddafi, K., 2018. Long-term trends and health impact of PM2. 5 and O3 in Tehran, Iran, 2006-2015. Environment international 114, 37-49.

23. Morsy, S., Dang, T. N., Kamel, M. G., Zayan, A. H., Makram, O. M., Elhady, M., Hirayama, K., Huy, N. T., 2018. Prediction of Zika-confirmed cases in Brazil and Colombia using Google Trends. Epidemiology \& Infection 146(13), 1625-1627. 